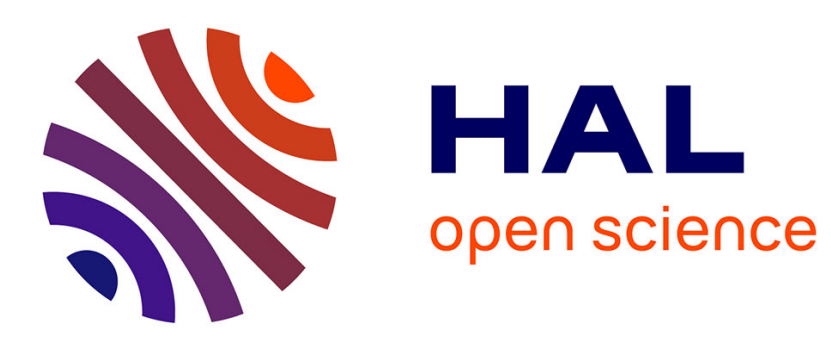

\title{
Aménagement de l'espace et problèmes fonciers au Praz de Lys (Haute-Savoie)
}

\author{
Michel Mietton
}

\section{To cite this version:}

Michel Mietton. Aménagement de l'espace et problèmes fonciers au Praz de Lys (Haute-Savoie). Revue de Géographie Alpine / Journal of Alpine Research, 1978, 66 (4), pp.409-420. 10.3406/rga.1978.2147 . hal-01165684

\section{HAL Id: hal-01165684 \\ https://univ-lyon3.hal.science/hal-01165684}

Submitted on 19 Jun 2015

HAL is a multi-disciplinary open access archive for the deposit and dissemination of scientific research documents, whether they are published or not. The documents may come from teaching and research institutions in France or abroad, or from public or private research centers.
L'archive ouverte pluridisciplinaire HAL, est destinée au dépôt et à la diffusion de documents scientifiques de niveau recherche, publiés ou non, émanant des établissements d'enseignement et de recherche français ou étrangers, des laboratoires publics ou privés. 


\section{Michel Mietton}

\section{Aménagement de l'espace et problèmes fonciers au Praz-de- Lys (Haute-Savoie) \\ In: Revue de géographie alpine. 1978, Tome 66 N4. pp. 409-420.}

\section{Résumé}

Résumé. - L'étude de l'aménagement foncier mené dans le cadre de l'implantation d'une station de ski au Praz de Lys (HauteSavoie) est particulièrement intéressante. II s'avère d'une part que les plus-values sur la terre sont le principal bénéfice que les montagnards peuvent retirer de cette opération. En second lieu, les intérêts particuliers y sont d'autant plus pressants qu'il s'agit d'une création en moyenne montagne où les propriétés privées sont nombreuses. Enfin, les solutions proposées sont originales, non pas tant par un plan d'occupation des sols qui privilégie les terrains communaux, mais par un mécanisme de transfert des coefficients inusité auparavant, visant tout à la fois à plus de justice et à une meilleure préservation du milieu.

\section{Abstract}

Summary. - Research on current land-parcelling meant for the selting up of a skiing-resort at Le Praz de Lys (Upper Savoy) has proved highly interesting. It turns out first of all that the unearned increments of the land constitute the main profit for mountain people to derive from such an enterprise. Secondly, if private interests loom large, they do all the more so as the resort is to be created in mid-altitude, where private estates generally abound. Lastly, the alternatives which have been put forward are original because they imply not so much a division of the land which is largely in favour of the Common Land as a mechanism of transferring of the rights so far never resorted to, whose aim is to improve both justice and the protection of our natural environment.

Citer ce document / Cite this document :

Mietton Michel. Aménagement de l'espace et problèmes fonciers au Praz-de-Lys (Haute-Savoie). In: Revue de géographie alpine. 1978, Tome $66 \mathrm{~N}^{\circ} 4$. pp. 409-420.

doi : 10.3406/rga.1978.2147

http://www.persee.fr/web/revues/home/prescript/article/rga_0035-1121_1978_num_66_4_2147 


\section{Aménagement de l'espace et problèmes fonciers au Praz de Lys. (Haute-Savoie)}

RÉSUMÉ. - L'étude de l'aménagement foncier mené dans le cadre de l'implantation d'une station de ski au Praz de Lys (Haute-Savoie) est particulièrement intéressante.

Il s'avère d'une part que les plus-values sur la terre sont le principal bénéfice que les montagnards peuvent retirer de cette opération. En second lieu, les intérêts particuliers y sont d'autant plus pressants qu'il s'agit d'une création en moyenne montagne où les propriétés privées sont nombreuses.

Enfin, les solutions proposées sont originales, non pas tant par un plan d'occupation des sols qui privilégie les terrains communaux, mais par un mécanisme de transfert des coefficients inusité auparavant, visant tout à la fois à plus de justice et à une meilleure préservation du milieu.

SUMMARY. - Research on current land-parcelling meant for the selting up of a skiing-resort at Le Praz de Lys (Upper Savoy) has proved highly interesting.

It turns out first of all that the unearned increments of the land constitute the main profit for mountain people to derive from such an enterprise.

Secondly, if private interests loom large, they do all the more so as the resort is to be created in mid-altitude, where private estates generally abound.

Lastly, the alternatives which have been put forward are original because they imply not so much a division of the land which is largely

* Nous remercions le Secrétaire de la Mairie de Taninges, M. CAVET, de l'aide qu'il nous a apportée. 


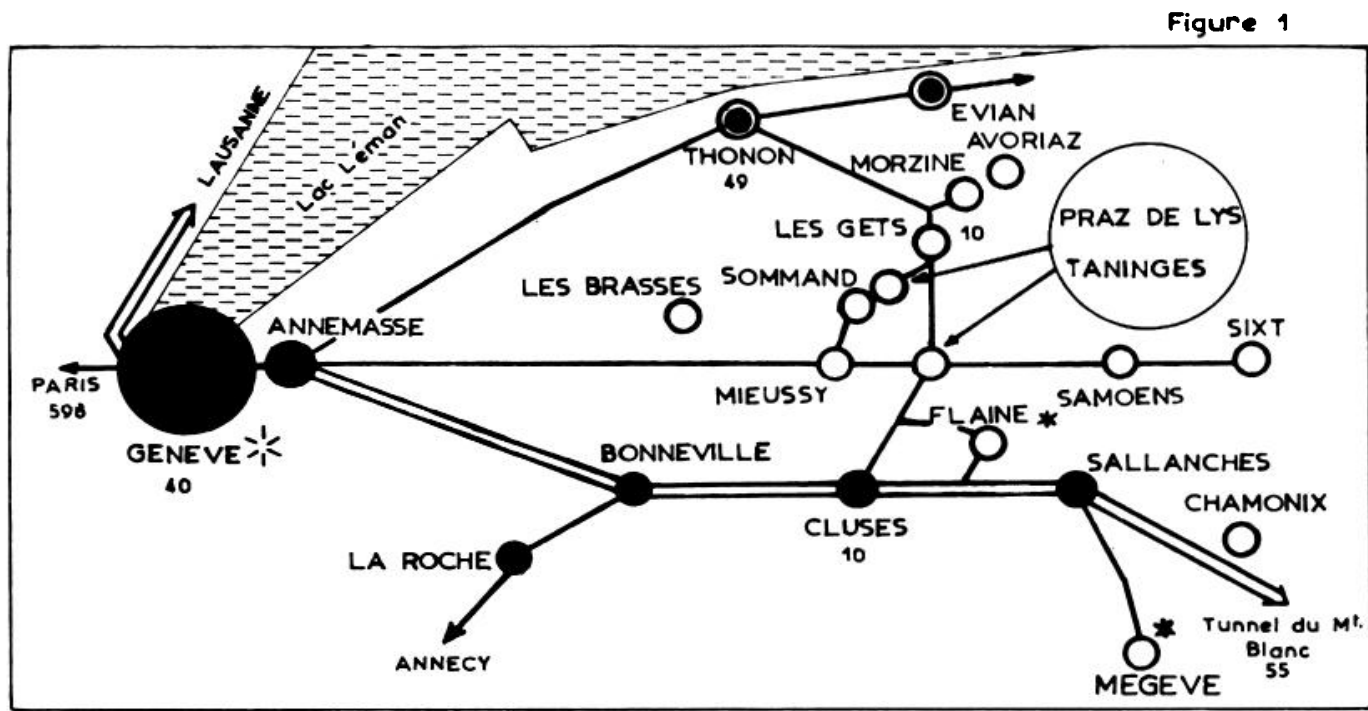

\section{LEGENDE}

- Villes principales

LA SITUATION DU PRAZ DE LYS

() Stations d’eré

Stations Ete-hiver

Routes principales

Autoroute

is Aeroport international

* Altipor

40 Kilométrage do partir de TANINOES

A. Cours d'eau et lac

an- Courbe de niveou el montagne

LE SITE DU PRAZ DE LYS
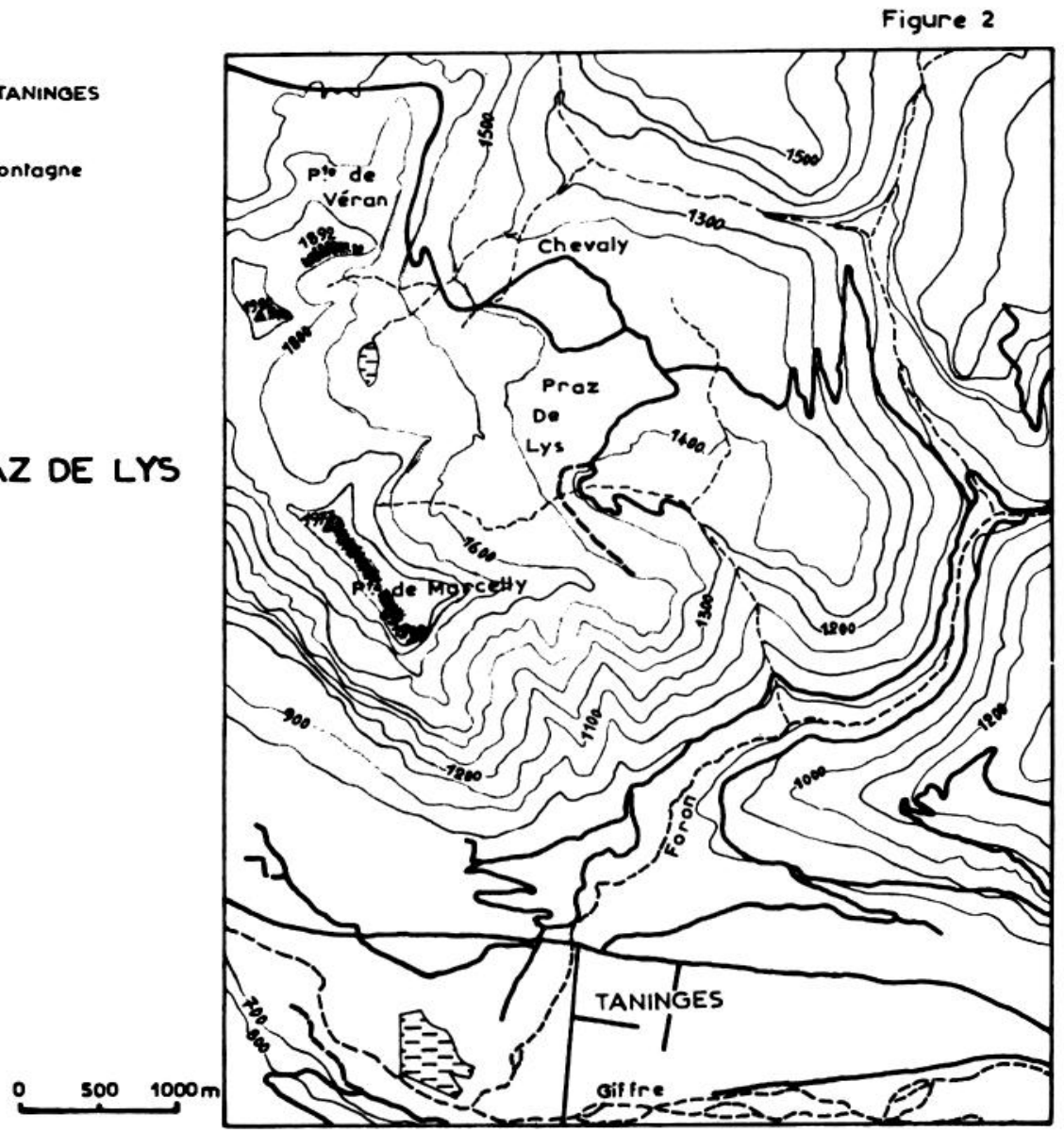
Le Chablais est en outre le massif préalpin le plus proche de Genève, de la région parisienne également et le Praz-de-Lys la première de ses stations (en venant de l'Ouest) avec celle plus modeste des Brasses, avant les grandes voisines de Samoëns, les Gets, Morzine-Avoriaz et Flaine.

Il s'agit bien là d'atouts suffisants pour prétendre assurer le succès d'une nouvelle station ; une fréquentation chaque année plus nombreuse en témoigne d'ailleurs aujourd'hui (doublement des recettes chaque année durant les quatre premières saisons de fonctionnement à Prazde-Lys - Sommand).

Dans le cadre de cette opération de Rénovation Rurale, la commune de Taninges bénéficie de 1,33 millions de francs de subventions pour la seule année 1970, soit trois fois plus qu'elle n'en avait perçu de 1950 à 1970 , et de 2,36 millions de prêts au taux de $4 \%$ sur vingt ans.

Aide d'autant plus appréciable qu'à cette date, les fonds de la Rénovation Rurale sont généralement destinés, sur le plan national, à des massifs beaucoup plus en difficulté que les Alpes du Nord: Vosges ou Massif Central par exemple, et qu'au niveau régional, la commune connaît alors une croissance d'ensemble certes limitée mais continue, s'appuyant sur un secteur industriel solide (industries alimentaires et industries du bois).

Enfin, à côté de ces avantages naturels et financiers, la commune de Taninges sait se doter, dès 1973, d'un outil supplémentaire pour assurer sa réussite : un plan d'occupation des sols (P.O.S.), cet outil étant ici profondément original dans la mesure où il intègre un mécanisme jusque-là inusité à notre connaissance : le transfert de coefficients d'occupation des sols (C.O.S.).

\section{I. - Le P.O.S. : outil de base d'une politique d'aménagement de l'espace.}

L'idée du P.O.S. ne s'impose pas d'emblée. Sa nécessité n'apparaît que progressivement lors des discussions, comme réponse aux différents problèmes fonciers.

\section{Les problèmes fonciers de l'aménagement :}

Le premier souci des aménageurs est évidemment de créer un réseau cohérent de pistes. Or, il apparaît très vite que les pistes devant être 
placées à l'Ouest où le relief est plus accusé, elles traverseront essentiellement des terrains particuliers ${ }^{1}$ (fig. 3).

Ces terrains étaient jusque-là peu intéressants : même en tant qu'alpages, ils recevaient de moins en moins de bêtes. A cela deux raisons : au lendemain de la deuxième guerre, la commune voit le nombre de ses agriculteurs diminuer, ceux-ci se tournant vers le secteur industriel à Taninges même et dans la vallée de l'Arve (Cluses); d'autre part, les agriculteurs restants préfèrent développer l'élevage dans la vallée où la commercialisation est plus facile.

Toutefois, dans le climat de discussions qui entoure les premières analyses, ces terrains prennent immédiatement de la valeur. Comment dès lors en obtenir la concession?

Quelques échanges entre propriétés communales et propriétés privées ont lieu. Puis une deuxième solution est envisagée : la location. Mais sur quelles bases : à l'hectare et par an? Il reste que la valeur d'un terrain skiable est difficile à établir, variant notamment en fonction du dynamisme des stations.

Parallèlement, il apparaît qu'il faut fixer les limites d'extension du complexe. L'expérience des stations voisines, les Gets par exemple, montre en effet combien il est coûteux d'assurer, entre autres, l'approvisionnement en eau. La mesure de la capacité des différentes sources réalisée au Praz-de-Lys conduit ainsi à la décision de limiter le nombre de lits entre 2500 et 3000 . Aller au-delà entraînerait inévitablement une augmentation des impôts locaux au cours des années à venir.

Ce chiffre peu imposant est tel cependant qu'il peut entraîner un «mitage » du plateau par dispersion de l'habitat.

Inversement, si l'on opère un regroupement, une petite superficie suffit. Mais dans ce cas, où opérer ce regroupement ?

Dans un souci d'équité, il s'agit en effet de ne pas favoriser un propriétaire par rapport à un autre au Praz-de-Lys ou d'avantager les propriétaires du Praz-de-Lys par rapport aux autres contribuables de la commune qui auront eux aussi à supporter les frais d'équipement de la station.

Il est clair que la seule solution à tous ces problèmes consiste alors à limiter les droits des particuliers au profit du plus grand nombre, au profit de la commune.

Cette solution, c'est le P.O.S.

1 Les propriétaires privés sont en outre nombreux dans cette moyenne montagne. Cinq seulement possèdent cinq hectares ou plus. 


\section{Le P.O.S. : un intérêt certain}

- En définissant des zones non aedificandi, le P.O.S. permet de créer des pistes ${ }^{2}$ et de conserver les forêts.

- En limitant partout ailleurs la construction, sauf au niveau d'un lotissement sur terrains communaux prévu pour 1500 lits, le P.O.S. freine la dispersion de l'habitat, ce qui a pour double conséquence de préserver encore les paysages, mais aussi de restreindre les travaux de viabilité à la charge de la commune.

Pour diminuer également ces travaux, le C.O.S., primitivement fixé à 0,050 , est ramené à 0,035 étant obtenu en faisant le rapport du nombre de lits restant à construire ${ }^{3}$ (ou plutôt de leur équivalent en $\mathrm{m}^{2}$ de plancher) à un certain nombre d'hectares, les zones non aedificandi étant évidemment exclues.

- Enfin, par une politique de la construction qui avantage les terrains communaux, le P.O.S. permet de mieux intéresser l'ensemble de la population à cette opération.

Souci de préservation du milieu, souci d'équité se retrouvent dans la mise en place du mécanisme de transfert de C.O.S.

\section{II. - Le transfert de C.O.S.: ou la recherche d'une mellleure intégration.}

\section{Un mécanisme original}

Différentes zones ont été distinguées sur le P.O.S. (fig. 3). Elles peuvent être regroupées en trois catégories :

- L'essentiel du plateau, environ 600 hectares sur 850 correspond aux zones non aedificandi.

Il s'agit principalement d'espaces boisés, de tourbières et de secteurs présentant des risques d'avalanches, l'ensemble disposant d'un C.O.S. nul.

2 Toutefois, ce réseau de pistes n'est pas totalement cohérent puisque l'une d'entre elles et le téléski de l'Hôtel recoupent la voie communale V.C. 228 reliant le centre du plateau au quartier du Pontet au Sud (Fig. 3). En l'absence d'aménagement, cette voie est barrée par une chaîne tout le temps que fonctionne le téléski.

3 Ce nombre de lits est de 1000 : sur un total de 3000 , il y en a 500 préexistants et 1500 prévus au lotissement. 
En revanche, les terrains affectés aux pistes et aux remontées mécaniques, également non aedificandi, bénéficient du C.O.S. de 0,035, celui-ci étant nécessairement transférables.

- Tout le reste du plateau est affecté d'un C.O.S. de 0,035. Ce C.O.S. est utilisable sur place à condition toutefois que la parcelle soit constructible, c'est-à-dire qu'elle soit viabilisée, de taille suffisante (le minimum constructible est de $2000 \mathrm{~m}^{2}$, dans ce cas le propriétaire ne dispose que de $70^{2}$ de plancher), et de forme adéquate (pas trop étroite par exemple).

- Mais le C.O.S. de 0,035 peut aussi être transféré vers trois sous-zones (UAa, UAa dit de la Savolière, UAb).

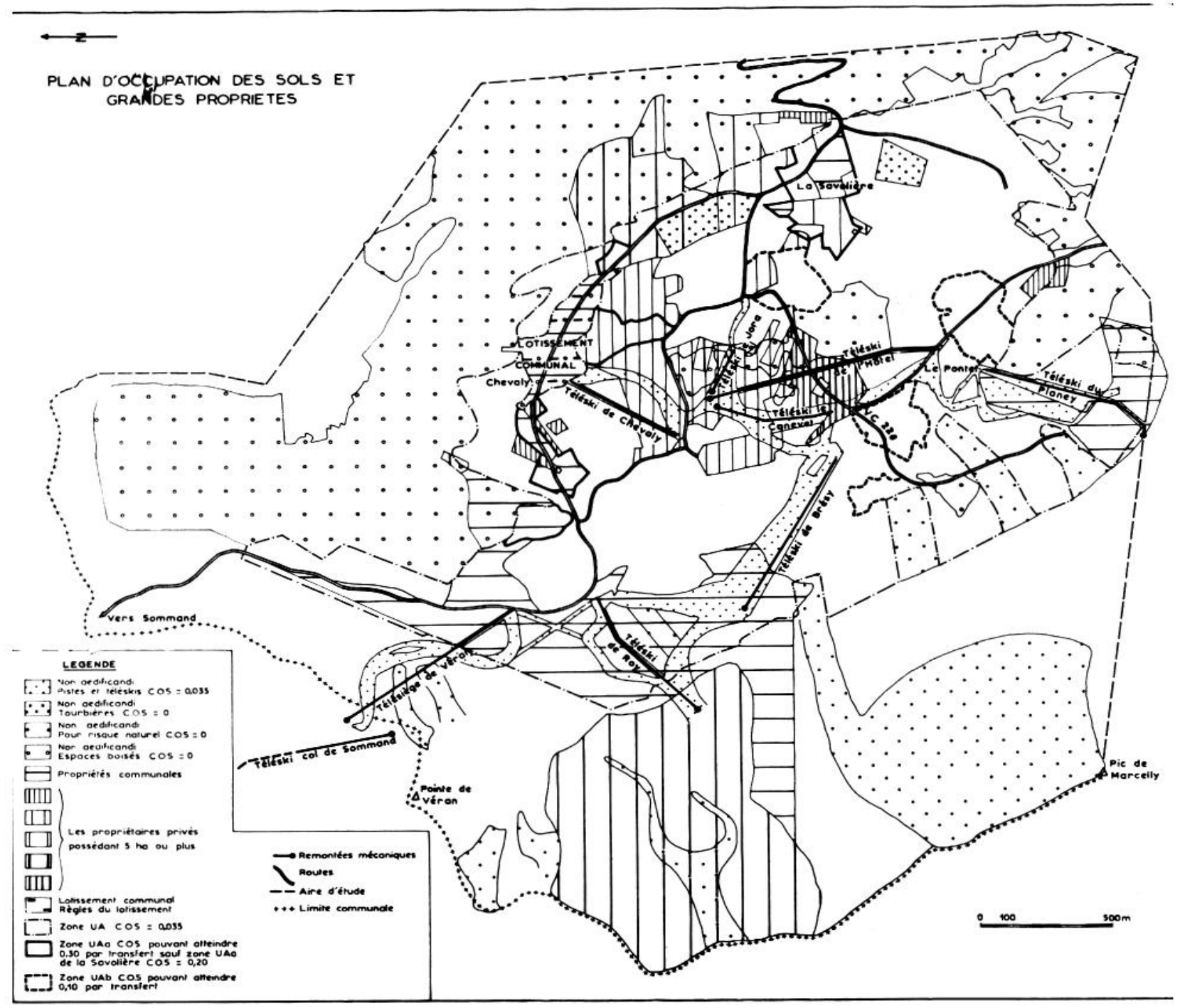

Fig. 3. - Plan d'Occupation des Sols et Grandes Propriétés 
Celles-ci sont bien entendu affectées du coefficient général $(0,035)$ mais ce dernier peut être porté, par transfert, respectivement à 0,30 , 0,20 et 0,10 . Précisons que le C.O.S. transférable est toujours égal à 0,035 .

Prenons l'exemple d'un propriétaire d'une parcelle de $1400 \mathrm{~m}^{2}$ située dans la zone UAa où le C.O.S. peut être porté à 0,30 . Le minimum constructible est ici comme en UAb de $1000 \mathrm{~m}^{2}$.

- Le propriétaire dispose d'emblée de :

$$
\frac{1400 \times 35}{1000}=49 \mathrm{~m}^{2} \mathrm{de} \text { plancher. }
$$

- Mais ses possibilités de construction sont de :

$$
\frac{1400 \times 30}{100}
$$

- S'il veut les utiliser, il lui faut donc acquérir par transfert en $\mathrm{m}^{2}$ de plancher :

$$
420-49=371 \mathrm{~m}^{2} \text { de plancher. }
$$

Soit une parcelle de :

$$
\frac{371 \times 1000}{35}
$$

Cette parcelle de $10600 \mathrm{~m}^{2}$, il peut soit la posséder et utiliser son C.O.S., soit l'acheter, soit acheter seulement le C.O.S. d'une ou plusieurs autres parcelles. Ces parcelles «vidées» de leur C.O.S. sont dès lors inconstructibles.

L'intérêt d'un tel mécanisme, nous allons le voir, est double.

\section{Le transfert de C.O.S. : un intérêt supplémentaire}

- L'inconstructibilité des parcelles vidées de leur C.O.S. faisant l'objet d'une inscription à la Conservation des hypothèques, ce mécanisme permet ainsi la sauvegarde définitive d'espaces verts. Il est intéressant de noter que le rapport entre le nombre de mètres carrés de plancher, construits grâce à ce procédé et le nombre de mètres carrés 1000

préservés est toujours de $\longrightarrow$, soit environ 30. Par exemple, pour 
d'espaces inconstructibles, le P.O.S. représente une effort courageux pour s'opposer aux intérêts particuliers et à une politique ultra-libérale incapable de sauvegarder le milieu naturel. Le mécanisme de transfert de C.O.S. permet à son tour de mieux faire accepter ce P.O.S. en atténuant les disparités dans les plus-values.

L'habileté des aménageurs a été, en outre, de tirer parti des inégalités du relief pour cacher le lotissement sur des terrains communaux (Chevaly) situés sur un gradin en contre-bas, au Nord du plateau (photo 2).

Le Praz de Lys diffère ainsi heureusement de la station de ski «classique », corps étranger de béton, plaqué contre la montagne, en désaccord complet avec la nature environnante. Cette absence d'urbanisation est le caractère fondamental retenu aujourd'hui par le touriste qui visite le Praz de Lys. Sera-t-il préservé ? Certes, tout le lotissement n'est pas encore construit : sur une dizaine d'hectares, il comprendra une soixantaine de parcelles, mais trente-cinq seront individuelles et une douzaine seulement consacrées aux commerces et à l'hôtellerie, le reste allant à des collectifs ou semi-collectifs de cinq étages au plus. Le caractère "camouflé 》 de ce centre doit donc demeurer.

Le lotissement communal n'est malheureusement pas le seul à pouvoir accueillir un habitat plus dense et il serait prématuré de conclure à une réussite totale.

\section{Les insuffisances de l'aménagement:}

Trois opérations de transfert seulement ont été réalisées à ce jour (Eté 1977) et les constructions sur terrains privés n'ont pas véritablement démarré. Les zones UAa et UAb sont toutefois appelées à en recevoir de nombreuses.

Il est en conséquence particulièrement important de préciser leur localisation de manière à imaginer leur impact sur le milieu et aussi de déterminer à qui profitent les forts coefficients.

- Il existe cinq zones UAa et quatre zones UAb représentant 21 , 5 et 21,2 hectares. Compte tenu des superficies et des coefficients respectifs, on peut estimer la surface constructible à $75000 \mathrm{~m}^{2}$ de plancher, ce qui est largement suffisant pour dégrader les paysages, d'autant que, si les zones UAb sont placées essentiellement dans la cuvette au sud du plateau, les secteurs UAa sont beaucoup plus en vue, au cour de la station.

- D'autre part, par confrontation du P.O.S. et de la carte des grandes propriétés (figure 3 ), il apparaît que le choix de ces zones s'est 
fait également de manière à satisfaire les plus gros possédants. A une exception près, tous se sont vus accorder des terrains UAa ${ }^{1}$. La politique « dirigiste » ayant conduit à une raréfaction du terrain à bâtir, celui-ci voit sa valeur majorée d'autant, mais la plus-value ne rapporte ici qu'à une minorité.

Il est vrai que la commune est, elle aussi, propriétaire de terrains UAa. C'est le cas notamment à l'Est du plateau, dans le secteur de la Savolière, où elle envisage de construire un Village-Vacances qui servirait ainsi à un tourisme social et renforcerait le caractère familial de la station. Il est vrai également que les secteurs UAa, à l'exception de la Savolière, sont accolés au lotissement communal et l'on peut penser que la commune essaiera d'acquérir ces terrains. Mais aura-t-elle les disponibilités financières à un moment où ses recettes lui servent à rembourser les prêts et alors qu'elle ne reçoit plus de subventions?

Quoiqu'il en soit, ces zones à coefficients élevés apparaissent trop nombreuses pour que l'intégration dans le site soit assurée dans l'avenir ${ }^{2}$.

Quant à l'intégration de la population de Taninges, elle n'est pas non plus ce que l'on souhaiterait. Les créations d'emplois sont insignifiantes : trois places de permanents et des postes saisonniers pour le reste. L'ensemble des commerces du lotissement, à une exception près, a été acheté par des gens venus de l'extérieur. On peut invoquer un manque de dynamisme des commerçants locaux, mais aussi les prix élevés pratiqués par la commune qui évidemment devait tirer le plus d'argent possible de la vente de ses terres.

\section{CONCLUSION}

La création d'une station de ski à Taninges n'a pas entraîné une véritable rénovation rurale, mais essentiellement une plus-value des terres au Praz de Lys.

Le mérite des aménageurs a été de s'attacher à faire profiter l'ensemble de la population de ce gonflement du capital foncier. Pour cela

\footnotetext{
1 On pourrait se demander si d'autres petits propriétaires n'ont pas reçu également des terrains UAa. En fait, la figure 3 , montre que la totalité de ces terrains appartient soit aux gros propriétaires, soit à la commune.

2 La pratique du ski de fond qui attire beaucoup de touristes aura aussi à souffrir de cette densification de l'habitat.
} 
ont été établis un P.O.S. privilégiant les terrains communaux et un mécanisme de transfert des C.O.S. visant tout à la fois à plus de justice entre les particuliers et à une préservation du milieu. Il s'agit là d'une expérience originale qui, sans oublier ses insuffisances, est à retenir pour d'autres opérations d'aménagement.

\section{BIBLIOGRAPHIE}

Bulletins communaux (1971):

- La rénovation rurale et ses répercussions sur la commune de Taninges en général, et le Praz de Lys en particulier.

- Où en est l'opération de rénovaton rurale ?

Règlement du plan d'occupation des sols (1973). Commune de Taninges. Secteur de Praz de Lys.

FAujas (A.) (1978). - UN EXEMPLE EN SAVOIE.

A Val-Morel, la station profite aussi aux montagnards.

Le Monde, 14 janvier.

RENARD (V.) (1976). - TROIS EXPERIENCES EN HAUTE-SAVOIE.

Les plans d'occupation des sols de Thônes, Saint-Jean-de-Sixt et Taninges. Laboratoire d'Economćtrie de l'Ecole Polytechnique, Paris.

Saint-Marc (Ph.) (1971). - SOCIALISATION DE LA NATURE, Paris, Stock.

VidAl et JENKINS (1970). - AMENAGEMENT D'UNE STATION DE MONTAGNE.

« PRAZ de LYS » (Haute-Savoie).

Considérations générales et Rapport justificatif. 